\title{
Non-Self-Adjoint Boundary Value Problems in Ordinary Differential Equations
}

\author{
Werner Greub ${ }^{1}$ and Werner C. Rheinboldt
}

(October 15, 1959)

\begin{abstract}
It is shown that the theory of non-self-adjoint linear ordinary differential equations can be simplified and unified if, instead of specifying linear boundary conditions in the conventional way, one merely specifies the linear subspace determined by the boundary conditions. If this is done, the corresponding linear space of the adjoint problem is the orthogonal complement of the original space with respect to the scalar product defined by the right-hand side of Green's boundary formula.
\end{abstract}

\section{Introduction}

The theory of boundary-value problems for ordinary differential equations of the form

$$
\sum_{\nu=0}^{n} a_{n-\nu}(t) u^{(\nu)}(t)=f(t)
$$

is now regarded as more or less complete. The selfadjoint case is presented in most textbooks owing to its importance in applications. A complete treatment of the non-self-adjoint problem is less common but may be found in Coddington, Levinson, ${ }^{2}$ and Ince. ${ }^{3}$ However, in these as well as in the original publications, e.g., Bôcher, ${ }^{4}$ the boundary conditions are stated in the form

$$
\begin{aligned}
& \omega_{\mu}\left(u^{\prime}(a), \ldots,\right.\left.u^{(n-1)}(a), u(b), \ldots, u^{(n-1)}(b)\right) \\
& \equiv \sum_{\nu=0}^{n-1}\left(A_{\mu \nu} u^{(\nu)}(a)+B_{\mu \nu} u^{(\nu)}(b)\right)=0 \\
& \quad(\mu=1, \ldots, m)
\end{aligned}
$$

where the $\omega_{\mu}$ are $m$ linearly independent linear functions in the $2 n$ dimensional number space $R^{2 n} \quad(0 \leqq m \leqq 2 n)$.

The $m$ linear equations $\omega_{\mu}=0 \quad(\mu=1, \ldots, m)$ define a $(2 n-m)$ dimensional linear subspace $\Omega \in R^{2 n}$ The set of solutions $u(t)$ of the problem (1)/(2) remains unchanged if the functions $\omega_{\mu}$ are replaced by another system of equations $\bar{\omega}_{\mu}=0 \quad(\mu=1, \ldots, m)$ which define the same subspace $\Omega \in R^{2 n}$.

\footnotetext{
1 The work was carried out while both authors were employed at the University of Maryland, the first in the Department of Mathematics, the second in the Institute for Fluid Dynamies and Applied Mathematics. The paper was prepared while both authors were affiliated with the National Bureau of Standards, the first working under a National Bureau of Standards contract with The A merican University. The authors' present addresses are, respectively: Mathematics Institute, University of Zurich, Zurich, Switzerland; and Computing Center, Institute, University of Zurich, Zu

2 Earl A. Coddington, Norman Levinson, Theory of ordinary differential ${ }^{2}$ Earl A. Coddington, Norman Levinson, Theory of ordinary

equations (McGraw-Hill Book Co., Inc., New York, N.Y., 1955).

M. B. Bocher, Applications and generalizations of the conception of adjoint ${ }^{4}$ M. Bôcher, Applications and generalizations of the
systems, Trans. Am. Math. Soc. 14, 403 to 420 (1913).
}

This fact causes an unnecessary complication for the theoretical investigation of such boundary value problems. It results especially in a somewhat artificial definition of the adjoint problem. These difficulties do not occur if one prescribes instead of the boundary conditions (2) only the subspace $\Omega$ of $R^{2 n}$, disregarding the manner in which $\Omega$ is represented. If this is done, the corresponding linear space of the adjoint problem is the orthogonal complement of the original space $\Omega$ with respect to a scalar product defined by the right-hand side of Green's boundary value formula.

It is shown in this article that the theory of the non-self-adjoint problems can be simplified and unified by a consequent use of these ideas and the methods of linear algebra.

\section{Green's Formula}

As usual we denote by $C^{k}(k=0,1, \ldots)$ the space of all real-valued ${ }^{5}$ functions $u(t)$ which possess $k$ continuous derivatives on a given closed interval $[a, b]$. Let $L[u]$ be the linear differential operator of order $n(\geqq 1)$ defined by

$$
L[u] \equiv \sum_{\nu=1}^{n} a_{n-\nu}(t) u^{(\nu)}(t)
$$

where $a_{n-\nu}(t) \in C^{\nu}, u(t) \in C^{n}$ and $a_{0}(t) \neq 0$ for $[a, b]$. To every function $u(t) \in C^{n-1}$ one can compute the $2 n$ real numbers

$$
u(a), u^{\prime}(a), \ldots, u^{(n-1)}(a), u(b), u^{\prime}(b), \ldots, u^{(n-1)}(b) .
$$

We regard these $2 n$ numbers as the components of a vector $\mathbf{u}$ of the $2 n$-dimensional linear number space $R^{2 n}$ and call it the boundary value vector of $u(t)$. The correspondence between the function $u(t)$ and its boundary value vector $\mathbf{u}$ is a linear mapping

5 The restriction to real-valued functions is not essential, as can be readily ascertained. 


$$
\mathbf{u}=\rho(u) \quad\left(u(t) \in C^{n-1}, \quad \mathbf{u} \in R^{2 n}\right)
$$

from $C^{n-1}$ onto $R^{2 n}$.

Let $\Omega$ be a given subspace of the $R^{2 n}$. Then the boundary value problem in consideration assumes the form

$$
L[u]=f(t), \quad \rho(u) \in \Omega,
$$

with given $f(t) \in C^{0}$. The corresponding homogeneous problem is

$$
L[u]=0, \quad \rho(u) \in \Omega .
$$

For any two functions $u(t), v(t) \in C^{n}$, the integral

$$
(L[u], v)=\int_{a}^{b} L[u] v d t
$$

is defined and by repeated partial integrations can be written as

$$
(L[u], v)=\left(u, L^{*}[v]\right)+[u, v]_{a}^{b},
$$

where

$$
\begin{gathered}
L^{*}[v]=\sum_{\nu=0}^{n}(-1)^{\nu}\left(a_{n-\nu}\right)^{(\nu)}, \\
{[u, v]_{a}^{b}=\left.\sum_{\nu=0}^{n-1} \sum_{\mu=\nu+1}^{n}(-1)^{\mu-\nu-1} u^{(\nu)}\left(a_{n-\nu} v\right)^{\mu-\nu-1}\right|_{a} ^{b} .}
\end{gathered}
$$

Equation (7) is the well-known Green's formula.

The linear differential operator $L^{*}$ is called the adjoint operator of $L$. 'The expression $[u, v]_{a}^{b}$ is a bilinear form in the two boundary value vectors $\rho(u), \rho(v)$. For arbitrary vectors

$$
\mathbf{x}=\left\{. ., \xi_{\nu}^{(a)}, \ldots ; . ., \xi_{\nu}^{(b)}, . .\right\}, \quad \mathbf{y}=\left\{\ldots, \eta_{\nu}^{(a)}, \ldots ; \ldots, \eta_{\nu}^{(b)}, \ldots\right\}
$$

of the $R^{2 n}$, this bilinear form can be written in the form

$$
\begin{aligned}
\varphi(\mathbf{x}, \mathbf{y}) & =\sum_{\nu=0}^{n-1} \sum_{\mu=0}^{n-\nu-1} \sum_{\lambda=\mu}^{n-\nu-1}(-1)^{\lambda}\left(\begin{array}{l}
\lambda \\
\mu
\end{array}\right) \\
& \cdot\left[a_{n-\nu-\lambda-1}^{(\lambda-\mu)}(b) \xi_{\nu}^{(b)} \eta_{\mu}^{(b)}-a_{n-\nu-\lambda-1}^{(\lambda-\mu)}(a) \xi_{\nu}^{(a)} \eta_{\mu}^{(a)}\right] .
\end{aligned}
$$

With this notation we get Green's formula,

$$
(L[u], v)=\left(u, L^{*}[v]\right)+\varphi(\rho(u), \rho(v)) .
$$

It is obvious that the correspondence $L \rightarrow L^{*}$ is linear. Furthermore, the adjoint operator to $L^{*}$ is again $L$. To show this, apply Green's formula to any $u, v \in C^{n}$ with $\rho(u)=\rho(v)=0$ :

$$
\begin{gathered}
(L[u], v)-\left(u, L^{*}[v]\right)=0 \\
\left(L^{*}[v], u\right)-\left(v, L^{* *}[u]\right)=0,
\end{gathered}
$$

which results in

$$
\left(L[u]-L^{* *}[u], v\right)=0
$$

(for all $u, v \in C^{n}$ with $\rho(u)=\rho(v)=0$ ) and thus leads to

$$
L[u]=L^{* *}[u]
$$

for all functions $u(t) \in C^{n}$. In the last step one has to use the same technique used in calculus of variations for the proof of Euler's condition.

The differential operator $L$ is said to be self-adjoint if $L^{*} \equiv L$. On comparing the forms (3) and (8) of $L$ and $L^{*}$ one sees immediately that $n$ must be even if the operator is self-adjoint.

In the case of a self-adjoint operator $L$ the lefthand side of (11) is skew-symmetric with respect to $u$ and $v$, and this implies that the bilinear form $\varphi(\mathbf{u}, \mathbf{v})$ is also skew-symmetric.

We will now show that $\varphi(\mathbf{x}, \mathbf{y})$ is not degenerate. To prove this it is sufficient to verify the following statement: If for a fixed vector $\mathbf{y} \in R^{2 n}$ we have $\varphi(\mathbf{x}, \mathbf{y})=0$ for every $\mathbf{x} \in R^{2 n}$, then it follows that $\mathbf{y}=0$.

We prove this first for a fixed vector $\mathbf{y}$ of the form

$$
\mathbf{y}_{a}=\left\{\eta_{0}^{(a)}, \ldots, \eta_{n-1}^{(a)}, 0, \ldots, 0\right\}
$$

or

$$
\mathbf{y}_{b}=\left\{0, \ldots ., 0, \eta_{0}^{(b)}, \ldots ., \eta_{n-1}^{(b)}\right\}
$$

taking $x$ correspondingly as $\mathbf{x}_{a}$ or $\mathbf{x}_{b}$, analogously defined. In this case the expression $\varphi\left(\mathbf{x}_{\alpha}, \mathbf{y}_{\alpha}\right)$ for $\alpha=a$ or $\alpha=b$ is a bilinear form in the two $n$-dimensional vectors $\left\{\xi_{0}^{(\alpha)}, \ldots . ., \xi_{n-1}^{(\alpha)}\right\}$ and $\left\{\eta_{0}^{(\alpha)}, \ldots ., \eta_{n-1}^{(\alpha)}\right\}$. Therefore, if $\varphi\left(\mathbf{x}_{\alpha}, \mathbf{y}_{\alpha}\right)=0$ holds for every $\mathbf{x}_{\alpha}$ and a fixed $\mathbf{y}_{\alpha}$, then in expression (10) the coefficient of every $\xi_{\nu}^{(\alpha)}$ must vanish separately. Thus

$$
\begin{aligned}
\sum_{\mu=0}^{n-\nu-1} \sum_{\lambda=\mu}^{n-\nu-1}(-1)^{\lambda}\left(\begin{array}{l}
\lambda \\
\mu
\end{array}\right) a_{n-\nu-\lambda-1}^{(\lambda-\mu)}(\alpha) \eta_{\mu}^{(\alpha)} & =0 \\
& (\nu=0, \ldots ., n-1) .
\end{aligned}
$$

For $\nu=n-1$ this has the form $a_{0}(\alpha) \eta_{0}^{(\alpha)}=0$, and since $a_{0}(\alpha) \neq 0$ it follows that

$$
\eta_{0}^{(\alpha)}=0
$$

For $\eta=n-2$ we get now

$$
a_{1}(\alpha) \eta_{0}^{(\alpha)}-a_{0}^{\prime}(\alpha) \eta_{0}^{(\alpha)}-a_{0}(\alpha) \eta_{1}^{(\alpha)}=-a_{0}(\alpha) \eta_{1}^{(\alpha)}=0
$$

and

$$
\eta_{1}^{(\alpha)}=0
$$

Continuing in this way, we ultimately show that

$$
\eta_{\mu}^{(\alpha)}=0 \quad(\mu=0, \ldots ., n-1)
$$

and thus $\mathbf{y}_{\alpha}=0$.

Now the proof is readily completed. From definition (10) it is seen that for arbitrary $\mathbf{x}=\mathbf{x}_{a}+\mathbf{x}_{b}$ and $\mathbf{y}=\mathbf{y}_{a}+\mathbf{y}_{b}$,

$$
\varphi(\mathbf{x}, \mathbf{y})=\varphi\left(\mathbf{x}_{b}, \mathbf{y}_{b}\right)-\varphi\left(\mathbf{x}_{a}, \mathbf{y}_{a}\right)
$$


For a fixed $\mathbf{y}$ this expression shall be zero for every $\mathbf{x} \in R^{2 n}$ and in particular for arbitrary vectors $\mathbf{x}=\mathbf{x}_{a}$ or $\mathbf{x}=\mathbf{x}_{b}$. Hence the two terms $\varphi\left(\mathbf{x}_{a}, \mathbf{y}_{a}\right)$ and $\varphi\left(\mathbf{x}_{b}, \mathbf{y}_{b}\right)$ have to vanish separately for every $\mathbf{x}_{a}$ and $\mathbf{x}_{b}$, respectively. In accordance with the first part of the proof, this is only possible for $\mathbf{y}_{a}=0$ and $\mathbf{y}_{b}=0$, i.e., $\mathbf{y}=0$.

\section{The Adjoint Problem}

The bilinear form $\varphi$ was shown to be nondegenerate. Accordingly, it can be employed to define "orthogonality" of vectors $\mathbf{x}, \mathbf{y} \in R^{2 n}$. We will say $\mathbf{x}$ and $\mathbf{y}$ are $\varphi$-orthogonal if $\varphi(\mathbf{x}, \mathbf{y})=0$. It should be observed that this $\varphi$-orthogonality is not necessarily symmetrical.

One can speak, in particular, of the $\varphi$-orthogonal space to $\Omega$ that is the space $\Omega^{\perp}$ of all vectors $\mathbf{y} \in R^{2 n}$ which are $\varphi$-orthogonal to all vectors $\mathbf{x} \in \Omega$.

For the dimension of $\Omega^{\perp}$ evidently holds

$$
\operatorname{dim} \Omega^{\perp}=2 n-\operatorname{dim} \Omega=m .
$$

Equation (13) implies

$$
\operatorname{dim}\left(\Omega^{\perp}\right)^{\perp}=\operatorname{dim} \Omega,
$$

and hence, together with $\left(\Omega^{\perp}\right)^{\perp} \in \Omega$, that

$$
\left(\Omega^{\perp}\right)^{\perp}=\Omega .
$$

It is worth while pointing out that the relation $\Omega \cap \Omega^{\perp}=0$ is not, in general, valid; there may exist a nonzero vector $\mathbf{x} \in \Omega$ which is $\varphi$-orthogonal to all vectors of $\Omega$.

With this terminology we define the adjoint boundary value problem of $\left(\pi_{N}\right)$ :

$$
\left(\pi_{N}^{*}\right) \quad L^{*}[u]=g(t), \quad \rho(u) \in \Omega
$$

with given $g(t) \in C^{0}$.

From $L^{* *}=L$ and (14), it follows that the adjoint problem of $\left(\pi_{N}^{*}\right)$ is again $\left(\pi_{N}\right)$.

In particular, $\left(\pi_{N}\right)$ will be termed self-adjoint ${ }^{6}$ if

$$
L^{*} \equiv L, \quad \Omega=\Omega^{\perp} .
$$

\section{Homogeneous Problems}

Consider the homogeneous problems $\left(\pi_{H}\right)$ and $\left(\pi_{H}^{*}\right)$. It is known from the theory of homogeneous linear differential equations that all solutions $u \in C^{n}$ of the equation

$$
L[u]=0
$$

form a $n$-dimensional function space $K$. The solutions $v \in C^{n}$ of $L^{*}[v]=0$ form another $n$-dimensional function space $K^{*}$. These two spaces $K$ and $K^{*}$ are mapped by the operator $\rho$ into two subspaces $\mathbf{K}=\rho(K)$ and $\mathbf{K}^{*}=\rho\left(K^{*}\right)$ of $R^{2 n}$.

${ }^{6}$ For self-adjoint problems Green's formula states

$$
(L[u], v)=(u, L[v]) \quad \text { for } \rho(u), \rho(v) \in \Omega
$$

Usually one uses this relation to define self-adjointness of $\left(\pi_{N}\right)$. The two definitions are equivalent as can be shown in a way similar to that used for the proof of $L^{*} \equiv L$.
In these subspaces $\mathbf{K}$ and $\mathbf{K}^{*}$ the operator $\rho$ has a unique inverse $\rho^{-1}$; hence, $K=\rho^{-1}(\mathbf{K})$ and $K^{*}=$ $\rho^{-1}\left(\mathbf{K}^{*}\right)$. This is immediately clear because $\rho(u)=0$ implies that all initial values $u^{(\nu)}(a) \quad(\nu=0,1, \ldots$, $n-1)$ are zero; hence $u(t)=0$ owing to the uniqueness theorem for the initial value problem of eq (16).

The operator $L$ and the bilinear form $\varphi(x, y)$ determine the $\phi$-orthogonal space $\mathbf{K}=\rho(K)^{\perp}$ of $\mathbf{K}$.

Theorem 1: The subspaces $\rho\left(K^{*}\right)$ and $\mathbf{K}^{\perp}=\rho(K)^{\perp}$ of $R^{2 n}$ are identical, i.e.,

$$
\mathbf{K}^{\perp}=\rho\left(K^{*}\right) .
$$

Proof: For any two functions $u(t) \in K, \quad v(t) \in K^{*}$ Green's formula gives $\varphi(\rho(u), \rho(v))=0$, which implies

$$
\rho\left(K^{*}\right) \in \rho(K)^{\perp}=\mathbf{K}^{\perp} .
$$

Since the mapping $\rho$ is regular in $K$ and $K^{*}$, it follows on the other hand that

and

$$
\operatorname{dim} \rho(K)=\operatorname{dim} \mathbf{K}=n
$$

$$
\operatorname{dim} \rho\left(K^{*}\right)=\operatorname{dim} \mathbf{K}^{*}=n .
$$

Thus, we find

$$
\operatorname{dim} \mathbf{K}^{\perp}=2 n-\operatorname{dim} \mathbf{K}=n,
$$

and the inclusion (18) must constitute equality.

To this point the boundary conditions lin $\left(\pi_{H}\right)$ and $\left(\pi_{H}^{*}\right)$ have not been used. To consider them now we introduce the space $A$ of all admissible functions of $\left(\pi_{H}\right)$, i.e., the space of all functions $u(t) \in C^{n}$ with $\rho(u) \epsilon \Omega$. Thus, the solutions of $\left(\pi_{H}\right)$ are exactly the functions

$$
u(t) \in A \cap K .
$$

For these functions the boundary value vectors are

$$
\rho(u) \in \Omega \cap \rho(K) .
$$

We handle $\left(\pi_{H}^{*}\right)$ in the same way. Let $A^{*}$ be the space of all $v(t) \in C^{n}$ for which $\rho(u) \in \Omega$. Then, the solutions of $\left(\pi_{H}^{*}\right)$ are exactly the functions

$$
v(t) \in A^{*} \cap K^{*} .
$$

In view of relation (17) the boundary value vectors of these functions are

$$
\rho(v) \in \Omega^{\perp} \cap \mathbf{K}^{\perp}=\Omega^{\perp} \cap \rho(K)^{\perp} .
$$

Thus, the number of linearly independent solutions of $\left(\pi_{H}\right)$ or $\left(\pi_{H}^{*}\right)$ is given by

$$
k=\operatorname{dim}(A \cap K)=\operatorname{dim}(\Omega \cap \rho(K))
$$

and

$$
k^{*}=\operatorname{dim}\left(A \cap K^{*}\right)=\operatorname{dim}\left(\Omega^{\perp} \cap \rho(K)^{\perp}\right),
$$

respectively. 
Theorem 2: The numbers $k$ and $k^{*}$ of linearly independent solutions of $\left(\pi_{H}\right)$ and $\left(\pi_{H}^{*}\right)$ are connected by the relation

$$
k^{*}=m-n+k \text {. }
$$

To prove this we use the following well-known lemma from linear algebra: then

Let $A$ be a linear space and $A_{1}, A_{2}$ two subspaces,

$$
\operatorname{dim} A_{1}+\operatorname{dim} A_{2}=\operatorname{dim}\left(A_{1} \cap A_{2}\right)+\operatorname{dim}\left(A_{1}+A_{2}\right),
$$

where $A_{1}+A_{2}$ is the linear closure of $A_{1}$ and $A_{2}$.

This relation is employed in a slightly different form. We introduce the orthogonal subspaces $A_{1}^{\perp} \in A$ and $A^{\perp} \in A$ of $A_{1}$ and $A_{2}$ with respect to a given (nondegenerate) bilinear form. Whence, we have

and

$$
\operatorname{dim} A_{1}^{\perp}=n-\operatorname{dim} A_{1}
$$

$\operatorname{dim}\left(A_{1}^{\perp} \cap A_{2}^{\perp}\right)=\operatorname{dim}\left(A_{1}+A_{2}\right)^{\perp}=n-\operatorname{dim}\left(A_{1}+A^{2}\right)$.

Thus (24) becomes

$\operatorname{dim} A_{2}-\operatorname{dim}\left(A_{1} \cap A_{2}\right)=\operatorname{dim} A_{1}^{\perp}-\operatorname{dim}\left(A_{1}^{\perp} \cap A_{2}^{\perp}\right)$.

In the present case,

$$
A=R^{2 n}, \quad A_{1}=\Omega, \quad A_{2}=\rho(K) ;
$$

therefore

$$
\operatorname{dim} A_{1}=2 n-m, \quad \operatorname{dim} A_{1}^{\perp}=m, \quad \operatorname{dim} A_{2}=n ;
$$

hence,

$$
n-\operatorname{dim}(\Omega \cap \rho(K))=m-\operatorname{dim}\left(\Omega^{\perp} \cap \rho(K)^{\perp}\right),
$$

which completes the proof.

\section{The Nonhomogeneous Problem}

Consider the nonhomogeneous problem $\left(\pi_{N}\right)$ together with its adjoint homogeneous problem $\left(\pi_{H}^{*}\right)$.

We shall prove the "alternative" theorem of the theory of systems of linear equations.

Theorem 3 (Alternative theorem): $\left(\pi_{N}\right)$ has a solution if and only if $(f, v)=0$ for all solutions $v(t)$ of $\left(\pi_{H}^{*}\right)$. This can be expressed in the form ${ }^{7}$

$$
L[A]=\left(A^{*} \cap K^{*}\right) \stackrel{\Perp}{ } .
$$

Here the symbol $\|$ denotes orthogonality in the function space $\mathrm{C}^{\circ}$ with respect to the scalar product $(f, g)$.

\footnotetext{
${ }^{7} L[A]$ is the subspace of $C^{\circ}$ containing all functions $L[u]$ with $u \in A$.
}

Proof: (1) Assume $\left(\pi_{N}\right)$ is solvable and $u(t)$ is a solution. Then, for any solution $v(t)$ of $\left(\pi_{H}^{*}\right)$, it follows from Green's formula that

$$
(f, v)=(L[u], v)=\left(u, L^{*}[v]\right)+\varphi(\rho(u), \rho(v)) .
$$

Since $L^{*}[v]=0$, then $\left(u, L^{*}[v]\right)=0$; and from $\rho(u) \in \Omega$ and $\rho(v) \in \Omega^{\perp}$ it follows that $\varphi(\rho(u), \rho(v))=0$. Thus it is seen that $(f, v)=0$.

(2) Assume that $(f, v)=0$ for all solutions of $\left(\pi_{H}^{*}\right)$. We shall show that $\left(\pi_{N}\right)$ has a solution.

From the existence theorem for ordinary differential equations it is known that the differential equation $L[u]=f$ must have at least one solution $u_{0}(t)$. For this function $u_{o}(t)$ and any solution $v(t)$ of $\left(\pi_{H}^{*}\right)$ we find from Green's formula that

$$
\begin{aligned}
0=(f, v)=\left(L\left[u_{0}\right], v\right) & =\left(u_{0}, L^{*}[v]\right)+\varphi\left(\rho\left(u_{0}\right), \rho(v)\right) \\
& =\varphi\left(\rho\left(u_{0}\right), \rho(v)\right) .
\end{aligned}
$$

Thus $\rho\left(u_{0}\right)$ is $\varphi$-orthogonal to all $\rho(v) \in \Omega^{\perp} \cap \rho(K)^{\perp}$ and

$$
\rho\left(u_{0}\right) \epsilon\left(\Omega^{\perp} \cap \rho(K)^{\perp}\right)=\Omega+\rho(K)
$$

where the + sign again denotes the linear closure of $\Omega$ and $\rho(K)$.

This inclusion states the existence of two vectors $\mathbf{u}_{1} \in \Omega, \quad \mathbf{u}_{2} \in \rho(K)$ such that $\rho\left(u_{0}\right)=\mathbf{u}_{1}+\mathbf{u}_{2}$. From the fact that $\rho$ has a unique inverse $\rho^{-1}$ in $\mathbf{K}$ it follows that there is exactly one function $u_{2}(t)=\rho^{-1}\left(\mathbf{u}_{2}\right) \in K$ for which $L\left[u_{2}\right]=0$ and $\rho\left(u_{2}\right)=\mathbf{u}_{2}$. We form a new function $u(t)=u_{0}(t)-u_{2}(t)$. This function $u(t)$ is a solution of $\left(\pi_{N}\right)$, for obviously $L[u]=f$ and $\rho(u)=$ $\rho\left(u_{0}\right)-\rho\left(u_{2}\right)=\mathbf{u}_{1} \in \Omega$.

Taking the orthogonal complement on both sides of (26) we find

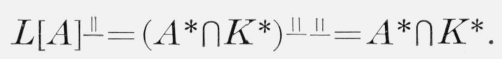

Thus we get the following

Corollary: If for a given function $v(t) \in C^{0}$,

$$
(v(t), L[u])=0 \quad \text { for every } \quad u(t) \in A,
$$

then $L^{*}[v]=0, \quad \rho(v) \in \Omega$ and in particular $v(t) \in C^{n}$.

\section{Green's Function}

The solution of the nonhomogeneous problem $\left(\pi_{N}\right)$-provided that it is solvable - is determined up to a solution of the homogeneous problem $\left(\pi_{H}\right)$. In order to obtain an explicit formula for the solution of $\left(\pi_{N}\right)$, it is necessary to assume an additional condition which uniquely determines the solution of $\left(\pi_{N}\right)$. Furthermore, it is desirable to modify $\left(\pi_{N}\right)$ in such a way that it is always solvable.

With these two aims in mind we introduce an orthonormalized basis in each of the spaces

$$
A \cap K \text { and } A^{*} \cap K^{*} .
$$


Lế

$$
u_{1}, u_{2}, \ldots, u_{k} \quad \text { and } v_{1}, v_{2}, \ldots, v_{k}
$$

be these two bases. With the functions $v_{v}(t)$ we define the new boundary value problem

$$
L[u]=f(t)-\sum_{\nu=1}^{k^{*}}\left(f(t), v_{\nu}(t)\right) v_{\nu}(t), \rho(u) \in \Omega .
$$

Owing to the orthonormality of the $v_{\nu}(t)$, the righthand side of the differential equation is orthogonal to all $v_{\nu}(t) \quad(\nu=1, \ldots, k)$, and thus to all solutions of $\left(\pi_{H}^{*}\right)$. Therefore (29) is solvable for every function $f(t) \in C^{0}$. To find an additional condition which uniquely determines the solution of problem (29), we recall the following fact: If $u(t)$ is a solution of (29), then

$$
u(t)+\sum_{\mu=1}^{k} \gamma_{\mu} u_{\mu}(t)
$$

is also a solution for any real coefficients $\gamma_{\mu}$. We can determine the $\gamma_{\mu}$ in such a way that

$$
\left(u(t)+\sum_{\mu=1}^{k} \gamma_{\mu} u_{\mu}(t), u_{\nu}(t)\right)=0 \quad(\nu=1, \ldots, k) .
$$

Owing to the orthonormality of the $u_{\mu}$, these relations are satisfied for

$$
\gamma_{\mu}=-\left(u(t), u_{\mu}(t)\right) \quad(\mu=1, \ldots, k) .
$$

This shows that there is always a solution of (29), which is orthogonal to all $u_{\mu}$ and thus to the whole space $A \cap K$; obviously, this solution is uniquely determined.

Consider instead of $\left(\pi_{N}\right)$ the following problem:

$$
\begin{aligned}
& L[u]=f(t)-\sum_{\mu=1}^{k^{*}}\left(f, v_{\mu}\right) v_{\mu}(t) \\
& \rho(u) \in \Omega \\
& \left(u(t), u_{\mu}(t)\right)=0 \quad(\mu=1, \ldots, k) .
\end{aligned}
$$

We know that this problem $\left(\hat{\pi}_{N}\right)$ has exactly one solution $u(t) \in C^{n}$ for every function $f(t) \in C^{0}$. The correspondence between $u(t)$ and $f(t)$ is denoted by an operator $G$, i.e.,

$$
u(t)=G[f(t)] .
$$

This operator is obviously linear. It will be shown that $G$ is an integral operator of the form

$$
u(t)=G[f(t)]=\int_{a}^{b} g(t, \tau) f(\tau) d \tau,
$$

where $g(t, \tau)$ is the so-called Green's function in the generalized sense.

To prove this, we consider functions $g(t, \tau)$ having the following properties:

(P 1) $g(t, \tau)$ is a continuous function of $t, \tau$ for $a \leqq t, \tau \leqq b$;
(P 2) For every arbitrary but fixed $\tau=\tau_{0}, a \leqq \tau_{0} \leqq b$, $g\left(t, \tau_{0}\right)$ possesses $n$ continuous derivatives for $a \leqq t \leqq \tau_{0}$ and $\tau_{0} \leqq t \leqq b$ such that

$$
\begin{gathered}
\frac{\partial^{\nu} g\left(\tau_{0}+0, \tau_{0}\right)}{\partial t^{\nu}}-\frac{\partial^{\nu} g\left(\tau_{0}-0, \tau_{0}\right)}{\partial t_{0}}=0 \quad(\nu=0,1, \ldots, n-2) \\
\frac{\partial^{n-1} g\left(\tau_{0}+0, \tau_{0}\right)}{\partial t^{n-1}}-\frac{\partial^{n-1} g\left(\tau_{0}-0, \tau_{0}\right)}{\partial t^{n-1}}=\frac{1}{a_{0}\left(\tau_{0}\right)} ;
\end{gathered}
$$

(P 3$)^{8}$ For every arbitrary but fixed $\tau=\tau_{0}$ in $a \leqq \tau_{0} \leqq b$,

$$
L_{t}\left[g\left(t, \tau_{0}\right)\right]=-\sum_{\mu=1}^{k^{*}} v_{\mu}(t) v_{\mu}\left(\tau_{0}\right)
$$

for $a \leqq t \leqq b$ but $t \neq \tau_{0}$;

$(\mathrm{P} 4)^{8}$ For every arbitrary but fixed $\tau=\tau_{0}$ in $a<\tau_{0}<b$,

$$
\rho_{t}\left(g\left(t, \rho_{0}\right)\right) \epsilon \Omega
$$

(P 5) For all functions $u_{\mu}(\mathrm{t}) \quad(\mu=1, \ldots, \mathrm{k})$ of the basis (28)

$$
\int_{a}^{b} g(t, \tau) u_{\mu}(t) \mathrm{d} t \equiv 0
$$

identically in $a \leqq \tau \leqq b$.

It shall be proved that there exists exactly one function $g(t, \tau)$ having these properties which satisfies (31).

Theorem 4: If there exists a function $g(t, \tau)$ possessing properties ( $\left.\begin{array}{ll}P & 1\end{array}\right)$ through $\left(\begin{array}{l}P \\ 5\end{array}\right)$, it is uniquely determined.

Proof: Let $g_{1}(t, \tau)$ and $g_{2}(t, \tau)$ be two functions which both possess properties (P 1) through (P 5). For an arbitrary but fixed $\tau=\tau_{0}$ in $a<\tau<b$ we define the function

$$
g_{0}(t)=g_{1}\left(t, \tau_{0}\right)-g_{2}\left(t, \tau_{0}\right) .
$$

Then, according to (P 3) it is seen that

$$
L\left[g_{0}(t)\right]=0 \quad \text { for } \quad a \leqq t \leqq b, \quad t \neq \tau_{0} .
$$

In view of (P 2), $g_{0}(t) \in C^{n-1}$; hence it follows from(32) that the jump in the $(n-1)$-th derivative of $g_{0}(t)$ at $t=\tau_{0}$ must be zero. Thus, $g_{0}(t) \in C^{n-1}$ and in view of $(32), g_{0}(t) \in K$. From $(\mathrm{P} 4)$ it follows that $g_{0}(t) \in A$; therefore, $g_{0}(t) \in K \cap A$. On the other hand, it can be concluded from $(\mathrm{P} 5)$ that $g_{0}(t) \in(K \cap A) \stackrel{\Perp}{ }$. This is possible only if $g_{0}(t) \equiv 0$ for $a \leqq t \leqq b$. Because $\tau_{0}$ was arbitrarily chosen in the open interval $a<\tau<b$, this is equivalent to the statement $g_{1}(t, \tau)=g_{2}(t, \tau)$ for $a \leqq t \leqq b, \quad a<\tau<b$, and in view of (P 1$)$ for the whole $a \leqq t, \tau \leqq b$. ${ }^{8}$ The subscripts $t$ in $L_{t}[g]$ and $\rho_{t}(g)$ denote that the differential operator $L$ and
the mapping $\rho$ operate on $g(t, \tau)$ regarded as a function of $t$. 
To state the existence of $g(t, \tau)$ we first prove the following:

Lemma 1: There exists at least one function $h(t, \tau)$ which possesses properties (P 1), ( $\left.P_{2}\right),\left(\begin{array}{l}P \\ 3\end{array}\right)$, and (P 5).

Proof: For fixed arbitrary $\tau$ in $a<\tau<b$, consider the ordinary differential equation

$$
L_{t}[g(t, \tau)]=-\sum_{\mu=1}^{k^{*}} v_{\mu}(t) v_{\mu}(\tau)
$$

and the two sets of initial values

$$
\left.\frac{\partial^{v} g(t, \tau)}{\partial t^{v}}\right|_{t=\tau}=0 \quad(\nu=0,1, \ldots, n-1)
$$

and

$$
\left.\frac{\partial^{n-1} g(t, \tau)}{\partial t^{n-1}}\right|_{t=\sigma}=\frac{1}{a_{0}(\tau)} .
$$

Both initial value problems (33)/(34a) and (33)/(34b) possess unique solutions $h_{1}(t, \tau)$ and $h_{2}(t, \tau)$, respectively. These solutions $h_{1}$ and $h_{2}$ are continuous functions of $t, \tau$ in the whole square, $a \leqq t, \tau \leqq b$. Furthermore, they possess $n$ continuous derivatives with respect to $t$ in this square. Accordingly, the function

$$
h_{3}(t, \tau)=\left\{\begin{array}{lll}
h_{1}(t, \tau) & \text { for } & a \leqq t \leqq \tau \\
h_{2}(t, \tau) & \text { for } & \tau \leqq t \leqq b
\end{array}\right.
$$

obviously possesses properties ( $\mathrm{P} 1),(\mathrm{P} 2)$, and (P 3).

We define the coefficient-functions

$$
c_{\mu}(\tau)=\left(h_{3}(t, \tau), u_{\mu}(t)\right) \quad(\mu=1, . ., k),
$$

which in view of the continuity properties of $h_{3}(t, \tau)$ are continuous functions of $\tau$. Hence, the function

$$
h(t, \tau)=h_{3}(t, \tau)-\sum_{\mu=1}^{k} c_{\mu}(\tau) u_{\mu}(t)
$$

evidently satisfies properties (P 1), ( $\mathrm{P} 2$ ), and (P 3). Furthermore (P 5) obtains, since

$$
\left(h(t, \tau), u_{\mu}(t)\right)=\left(h_{3}(t, \tau), u_{\mu}(t)\right)-c_{\mu}(\tau)=0
$$

$$
(\mu=1, \ldots, k),
$$

owing to the orthonormality of the $u_{\mu}(t)$.

Lemma 2: There exists a linear transformation which maps every vector $\mathbf{x} \in \Omega+\rho(K)$ into one vector $\mathrm{y} \in \rho(K)$ such that

$$
\left(\rho^{-1}(\mathbf{y}), u_{\mu}(t)\right)=0 \quad(\mu=1, \ldots, k) .
$$

Proof: Let $\mathbf{x} \in \Omega+\rho(K)$ be given. Then there exist at least two vectors $\mathbf{x}_{1}, \mathbf{x}_{2}$ for which

$$
\mathbf{x}=\mathbf{x}_{1}+\mathbf{x}_{2} \quad \mathbf{x}_{1} \in \Omega \quad \mathbf{x}_{2} \in \rho(K) .
$$

$\mathbf{x}_{1}$ and $\mathbf{x}_{2}$ are determined to within a vector belonging to the intersection $\Omega \cup \rho(K)$. With coefficients

$$
c_{\mu}=\left(\rho^{-1}\left(\mathbf{x}_{2}\right), u_{\mu}(t)\right) \quad(\mu=1, \ldots, k),
$$

the following new vectors are defined:

$$
\mathbf{y}_{1}=\mathbf{x}_{1}+\sum_{\mu=1}^{k} c_{\mu} \rho\left(u_{\mu}\right), \mathbf{y}_{2}=\mathbf{x}_{2}-\sum_{\mu=1}^{y} c_{\mu} \rho\left(u_{\mu}\right) .
$$

Since

$$
\sum_{\mu=1}^{k} c_{\mu} \rho\left(u_{\mu}\right) \in \Omega \cap \rho(K)
$$

then

$$
\mathbf{x}=\mathbf{y}_{1}+\mathbf{y}_{2} \quad \mathbf{y}_{1} \in \Omega \quad \mathbf{y}_{2} \in \rho(K),
$$

and, furthermore, in view of the orthonormality of the $u_{\mu}$,

$$
\begin{array}{r}
\left(\rho^{-1}\left(\mathbf{y}_{2}\right), u_{\mu}\right)=\left(\rho^{-1}\left(\mathbf{x}_{2}\right), u_{\mu}\right)-\sum_{\nu=1}^{k} c_{v}\left(\rho^{-1} \rho\left(u_{v}\right), u_{\nu}\right) \\
=\left(\rho^{-1}\left(\mathbf{x}_{2}\right), u_{\mu}\right)-c_{\mu}=0 \quad(\mu=1, \ldots, k) .
\end{array}
$$

The two vectors $\mathbf{y}_{1}$ and $\mathbf{y}_{2}$ are uniquely determined by conditions (36) and (37). This is rather obvious; for any other decomposition $\mathbf{x}=\mathbf{y}_{1}^{\prime}+\mathbf{y}_{2}^{\prime}$ of $\mathbf{x}$ which satisfies (36) and (37) it follows that $\rho^{-1}\left(\mathbf{y}_{2}-\mathbf{y}_{2}^{\prime}\right) \in A \cap K$. However, the relations

$$
\left(\rho^{-1}\left(\mathbf{y}_{2}-\mathbf{y}_{2}^{\prime}\right), u_{\mu}\right)=0 \quad(\mu=1, \ldots, k)
$$

obtain only if $\mathbf{y}_{2}=\mathbf{y}_{2}^{\prime}$ which then implies $\mathbf{y}_{1}=\mathbf{y}_{1}^{\prime}$.

Hence, it has been shown that we can associate with every $\mathbf{x} \in \Omega+\rho(K)$ exactly one vector $\mathbf{y}_{2} \in \rho(K)$ which fulfills (36) and (37). This was the statement of the lemma.

It is necessary at this point to obtain an extension of Green's formula for functions having discontinuity properties similar to $(\mathrm{P} 2)$. Let the function $y(t)$ possess the following properties:

a) $y(t)$ possesses $n$ continuous derivatives for $a \leqq t \leqq \tau, \quad \tau \leqq t \leqq b$, where $\tau$ is a certain fixed point.

b) $y^{(\nu)}(\tau+0)-y^{(\nu)}(\tau-0)=0 \quad(\nu=0,1, \ldots, n-2)$,

$$
y^{(n-1)}(\tau+0)-y^{(n-1)}(\tau-0)=c,
$$

where $c$ is a certain fixed constant.

For any arbitrary chosen $v(t) \in C^{n}$, it can be shown by repeated partial integration that

$$
(L[y], v)-\left(y, L^{*}[v]\right)=\varphi(\rho(y), \rho(v))-c a_{0}(\tau) v(\tau) .
$$

This is the generalized Green's formula.

Theorem 5: There exists a function $g(t, \tau)$ which possesses the five properties ( $\mathrm{P} 1)$ through ( $\mathrm{P} 5)$.

Proof: Let $h(t, \tau)$ be the function which has properties (P 1), ( $\mathrm{P} 2$ ), $\mathrm{P}$ 3), and ( $\mathrm{P}$ 5). For every arbitrary but fixed $\tau$ in $a<\tau<b$, insert $h(t, \tau)$ and the basic functions $v_{\nu}(t) \quad(\nu=1, \ldots, ., k)$ in the generalized Green's formula:

$$
\begin{aligned}
\left(L_{t}[h], v_{\nu}\right) & =\left(h, L^{*}\left[v_{\nu}\right]\right)+\varphi\left(\rho_{t}(h), \rho\left(v_{\nu}\right)\right)-v_{\nu}(\tau) \\
& =\varphi\left(\rho_{t}(h), \rho\left(v_{\nu}\right)\right)-v_{\nu}(\tau) \quad\left(\tau=1, \ldots, k^{*}\right) .
\end{aligned}
$$


On the other hand, for $a \leqq t \leqq b, \quad t \neq \tau$, according to (P 3),

$$
\left(L_{t}[h], v_{\nu}\right)=-\sum_{\mu=1}^{k^{*}}\left(v_{\mu}(t), v_{\nu}(t)\right) v_{\nu}(\tau)=-v_{\nu}(\tau),
$$

and in view of the orthonormality of the $v_{\nu}(t)$

$$
\varphi\left(\rho_{t}(h), \rho\left(v_{\nu}\right)\right)=0 \quad\left(\nu=1, \ldots, k^{*}\right) .
$$

This is equivalent to

$$
\rho_{t}(h) \in\left(\Omega^{\perp} \bigcup_{\rho}(K)^{\perp}\right)^{\perp}=\Omega+\rho(K) .
$$

We now consider the (continuous) linear transformation from $\Omega+\rho(K)$ into $\rho(K)$ which exists by lemma 2. Let $\mathbf{h} \in \rho(K)$ with

$$
\left(\rho^{-1}(\mathbf{h}), u_{\mu}\right)=0 \quad(\mu=1, \ldots, k)
$$

be the image vector of $\rho_{t}(h)$ under this transformation. Then $\mathbf{h}$ is a continuous function of $\tau$ for $a \leqq \tau \leqq b$. This is obvious because $\rho_{t}(h)$ depends continuously on $\tau$, and the correspondence between $\rho_{t}(h)$ and $\mathbf{h}$ is continuous. Let $\mathbf{x}_{1}, \ldots, \mathbf{x}_{n}$ be a basis of $\rho(K)$ and

$$
\mathbf{h}=\sum_{\mu=1}^{n} d_{\mu}(\tau) \mathbf{x}_{\mu}
$$

It is clear that the coefficients $d_{\mu}(\tau)$ are continuous functions of $\tau$ for $a \leqq \tau \leqq b$. Hence, the (uniquely determined) function

$$
\hat{h}(t, \tau)=\rho^{-1}(\mathbf{h})=\sum_{\mu=1}^{n} d_{\mu}(\tau), \quad \rho^{-1}\left(\mathbf{x}_{\mu}\right) \in K
$$

is continuous in both variables $t$ and $\tau$ for $a \leqq t, \tau \leqq b$.

We define the function

$$
g(t, \tau)=h(t, \tau)-\hat{h}(t, \tau) .
$$

Then $g(t, \tau)$ obviously possesses properties ( $\mathrm{P} 1$ ), (P 2), and (P 3). (P 4) follows from

$$
\rho_{t}(g)=\rho_{t}(h)-\mathbf{h} \in \Omega .
$$

Finally, as a consequence of (39),

$$
\left(g(t, \tau), u_{\mu}(t)\right)=\left(h(t, \tau), u_{\mu}(t)\right)-\left(\hat{h}(t, \tau), u_{\mu}(t)\right)=0
$$

for $\mu=1, \ldots, k$, since $h(t, \tau)$ fulfills (P 5$)$.

Thus $g(t, \tau)$ possesses properties (P1) through (P 5). This proves the theorem.

Now we are in a position to prove the following final theorem:

Theorem 6: Let $g(t, \tau)$ be the uniquely determined function with the properties (P1) through (P5); then

$$
u(t)=G[f(t)]=\int_{a}^{b} g(t, \tau) f(\tau) d \tau
$$

for every given $f(\tau) \in C^{\circ}$, constitutes the (uniquely determined) solution of $\left(\hat{\pi}_{N}\right)$.

Proof: Let $v(t) \in A^{*}$ be an arbitrary function. For any fixed $\tau$ in $a<\tau<b$ we apply the generalized Green's formula (38) to $g(t, \tau)$ and $v(t)$. With regard to $(\mathrm{P} 4)$ and $\rho(v) \in \Omega^{\perp}$ it is seen that

$$
\left(L_{t}[g], v\right)=\left(g, L^{*}[v]\right)-v(\tau)
$$

thus, in view of (P3),

$$
\left(L_{t}[g], v\right)=-\sum_{\nu=1}^{k^{*}}\left(v, v_{v}\right) v_{v}(\tau)=\left(g, L^{*}[v]\right)-v(\tau)
$$

for $a \leqq t \leqq b, \quad a<\tau<b, \quad t=\tau$. We multiply this equation by a continuous function $f(\tau)$ and integrate over $\tau$. Because $g$ is a continuous function of $t$ and $\tau$, it follows that

$$
-\sum_{\nu=1}^{k^{*}}\left(v, v_{v}\right)\left(v_{v}, f\right)=\left(G[f], L^{*}[v]\right)-(f, v) .
$$

For $f(t)$ we consider the boundary value problem (29) which is solvable no matter how $f(t)$ was chosen. Let $u_{1}(t)$ be a solution. For the given function $v(t) \in A^{*}$ we obtain

$$
\left(L\left[u_{1}\right], v\right)=(f, v)-\sum_{v=1}^{k^{*}}\left(f, v_{v}\right)\left(v_{v}, v\right),
$$

and thus together with (40)

$$
\left(L\left[u_{1}\right], v\right)=\left(G[f], L^{*}[v]\right) .
$$

Applying Green's formula (11) to the left-hand side we conclude that

$$
\left(L^{*}[v], u_{1}\right)=\left(G[f], L^{*}[v]\right)-\varphi\left(\rho\left(u_{1}\right), \rho(v)\right),
$$

and because $\varphi\left(\rho\left(u_{1}\right), \rho(v)\right)=0$, then

$$
\left(G[f]-u_{1}, L^{*}[v]\right)=0 .
$$

This relation is valid for every function $v(t) \in A^{*}$. Hence, it follows from the corollary to the alternative theorem that $G[f]-u_{1} \in C^{n}$; thus, $u(t)=G[f]$ $\in C^{n}$. Furthermore, it follows that

$$
L\left[G[f]-u_{1}\right]=0, \quad \rho\left(G[f]-u_{1}\right) \in \Omega,
$$

which is equivalent to

$$
\begin{aligned}
& L[G[f]]=L\left[u_{1}\right]=f-\sum_{\nu=1}^{k^{*}}\left(f, v_{v}\right) v_{v}, \\
& \rho(G[f]) \in \Omega .
\end{aligned}
$$

Finally, it follows from (P5) and (P1) that

$$
\left(u(t), u_{\mu}(t)\right)=0 \quad(\mu=1, \ldots, k) .
$$

In applying theorem 6 to the adjoint problem $\left(\hat{\pi}_{N}{ }^{*}\right)$ we confirm that for any $f_{1}(t) \in C^{\mathrm{o}}$ the uniquely 
determined solution $v(t)$ of $\left(\hat{\pi}_{\mathrm{N}}{ }^{*}\right)$ is given by

$$
v(t)=G^{*}\left[f_{1}\right]=\int_{a}^{b} g^{*}(t, \tau) f^{1}(\tau) d \tau .
$$

We will show that the integral operator $G^{*}$ is the adjoint operator of $G$, i.e.,

$$
\left(G[f], f_{1}\right)=\left(f, G^{*}\left[f_{1}\right]\right)
$$

for all $f_{1}, f \in C^{\circ}$.

Let $v(t)=G^{*}\left[f_{1}\right]$, i.e.,

$$
\begin{aligned}
& L^{*}\left[G^{*}\left[f_{1}\right]\right]=f_{1}-\sum_{\nu=1}^{k}\left(f_{1}, u_{v}\right) u_{v} \\
& \rho\left(G^{*}\left[f_{1}\right]\right) \in \Omega^{\perp} ; \quad\left(G^{*}\left[f_{1}\right], v_{\mu}\right)=0 \quad\left(\mu=1, \ldots, k^{*}\right) .
\end{aligned}
$$

Washington, D.C.

(Paper 64B2-24)
With another continuous function $f(t)$ we find from Green's formula (11),

$$
\begin{aligned}
\left(G[f], f_{1}\right)= & \left(G[f], L^{*}\left[G^{*}\left[f_{1}\right]\right]\right)+\sum_{\nu=1}^{k}\left(f_{1}, u_{v}\right)\left(G[f], u_{v}\right) \\
= & \left(L[G[f]], G^{*}\left[f_{1}\right]\right)+\varphi\left(\rho(G[f]), \rho\left(G^{*}\left[f_{1}\right]\right)\right) \\
& +\sum_{\nu=1}^{k}\left(f_{1}, u_{v}\right)\left(G[f], u_{v}\right) \\
= & \left(f, G^{*}\left[f_{1}\right]\right)+\sum_{\mu=1}^{k^{*}}\left(f, v_{\mu}\right)\left(v_{\mu}, G^{*}\left[f_{1}\right]\right) \\
& +\varphi\left(\rho(G[f]), \rho\left(G^{*}\left[f_{1}\right]\right)\right)+\sum_{\nu=1}^{k}\left(f_{1}, u_{v}\right)\left(G[f], u_{v}\right) .
\end{aligned}
$$

Since $G\left[f_{1}\right]$ and $G^{*}\left[f_{1}\right]$ are solutions of $\left(\hat{\pi}_{N}\right)$ and $\left(\hat{\pi}_{N} *\right)$, respectively, the last three quantities must vanish, which leads to (42).

From (42) follows for the corresponding Green's functions,

$$
g^{*}(t, \tau)=g(\tau, t) .
$$

